\title{
Evaluation of a general toxicity study incorporating phototoxicity assessments in Sprague-Dawley rats
}

\author{
Yutaka Yonezawa1,2, Taishi Miyashita1', Hiroko Ashizawa1', Kazuto Hashimoto1, \\ Hiroaki Nejishima1 ${ }^{1}$ and Haruko Ogawa ${ }^{3}$
}

\author{
'Pharmacokinetics and Safety Department Drug Research Center Kaken Pharmaceutical Co., Ltd., \\ 301, Gensuke, Fujieda, Shizuoka 426-8646, Japan \\ ${ }^{2}$ United Graduate School of Veterinary Sciences, Gifu University, 1-1 Yanagido, Gifu, Gifu 501-1193, Japan \\ ${ }^{3}$ Diagnostic Center for Animal Health and Food Safety, Obihiro University of Agriculture and Veterinary Medicine, \\ 2-11 Inada, Obihiro, Hokkaido 080-8555, Japan
}

(Received November 9, 2016; Accepted December 27, 2016)

\begin{abstract}
Previously, we showed that phototoxicity assessments in Sprague-Dawley (SD) rats can detect phototoxic potential to the same degree as those in guinea pigs. In this study, we examined whether phototoxicity assessments can be incorporated into general toxicology studies, using SD rats. Three phototoxic compounds were tested. Acridine and 8-methoxypsoralen (8-MOP) were transdermally administered, and 8-MOP and lomefloxacin were orally administered. The animals were allocated to three groups for each compound: single-dose, repeated-dose, and repeated-dose plus toxicokinetics (TK). The singledose group was irradiated with UV-A and UV-B after a single administration of the drug. The repeateddose and TK groups were irradiated after 8 days of repeated administration of the drug. Blood samples were also collected from the TK group on days 1 and 7 after administration. The phototoxic compounds resulted in skin reactions in all the groups, with no difference in the degree of skin reaction among the three groups. In the TK measurements, all of the phototoxic compounds were detected in the plasma samples, and the irradiation timing was close to the $\mathrm{T}_{\max }$. These results indicate that phototoxic potential could be evaluated in the TK group, and phototoxicity assessments could be incorporated into general toxicology studies. This reduces the number of studies and animals required, thus shortening the research and development period, and supporting the 3 Rs principle of animal experiments. The study also provides information regarding appropriate irradiation timings, differences between the sexes, and dose-response, in turn enabling the phototoxic risk of the compounds to be clearly evaluated.
\end{abstract}

Key words: Phototoxicity, Incorporating assessment, Sprague-Dawley (SD) rat, 8-MOP, Lomefloxacin, General toxicity study

\section{INTRODUCTION}

Phototoxicity is defined as an acute, light-induced tissue response to a photoreactive chemical, with phototoxic responses being clinically characterized by erythema and edema. In a clinical setting, the use of phototoxic drugs prevents patients from going outdoors during daytime, because these drugs are excited by exposure to sunlight and cause toxic reactions, resulting in erythema and edema (Diffey et al., 1983). Additionally, since the treatment of some skin diseases requires ultraviolet irradiation (Parrish et al., 1974), use of phototoxic drugs prevents patients from being given such treatments. Hence, it is very important to evaluate the phototoxic risk of compounds during drug development.

Phototoxicity studies are usually performed as independent studies. However, incorporating assessments with general toxicity studies has recently been recommended in the International Council for Harmonization of Technical Requirements for Pharmaceuticals for Human Use (ICH) guideline M3(R2) (2009) and the ICH guideline S2(R1) (2008). Additionally, the Guidance for Industry Photosafety Testing (2003) published by the Food and Drug Administration (FDA) specifies, "assessments of photoirritation may be incorporated into ongoing general toxicity studies in some circumstances."

Correspondence: Yutaka Yonezawa (E-mail: yonezawa_yutaka@kaken.co.jp) 
Toxicology studies incorporating genotoxicity (Hamada et al., 2001) and pharmacological safety (Luft and Bode, 2002) assessments have already been reported. These reports indicate that combining multiple studies shortens the research and development period and reduces the number of experiments and animals required. In addition, Hamada's report (Hamada et al., 2001) suggests that information concerning toxicokinetics and other diverse toxicity information can be obtained simultaneously from the same animals, which is beneficial for the comprehensive safety evaluation of chemicals. Thus, the ability to incorporate phototoxicity assessments into general toxicity studies could be of huge benefit for accelerating the testing of new drugs and reducing the number of animal experiments required during drug testing.

In a previous study (Yonezawa et al., 2015), we showed that phototoxicity assessments in Sprague-Dawley (SD) rats can detect phototoxic potential to the same degree as those in guinea pigs, which are typically used as an in vivo model (Morikawa et al., 1974). We used SD rats because the ICH guideline S10 (2013) states that animals should be irradiated at approximately the time of maximum concentration $\left(\mathrm{T}_{\max }\right)$, and therefore phototoxicity studies require the collection of pharmacokinetic (PK) data. In the early stages of drug development, PK data is commonly collected using mice and rats. However, if the phototoxicity study is performed using guinea pigs, additional PK data must be collected for guinea pigs in another experiment. SD rats are widely used to measure the PK profiles of drugs before phototoxicity studies, and therefore using SD rats for the phototoxicity study as well shortens the study period, and is in keeping with the 3Rs principle (Replacement, Reduction and Refinement) of animal experiments because it removes the need for an additional PK study. Additionally, SD rats are widely used in general toxicity studies, so it may be possible to incorporate phototoxicity assessments into general toxicity studies in SD rats.

In this study, we examined whether a phototoxicity assessment could be incorporated into a general toxicology study, using SD rats. We compared the phototoxic reactions in three groups-single-dose, repeated-dose, and repeated-dose plus toxicokinetics (TK) - to determine whether the repeated-dose and toxicokinetic evaluation had any influence on the phototoxic response. In addition, we evaluated whether phototoxic potential could be evaluated in the TK group.

\section{MATERIALS AND METHODS}

\section{Chemicals}

Three known phototoxic compounds were used as model compounds in this study: 8-methoxypsoralen (8-MOP), acridine, and lomefloxacin. 8-MOP was purchased from Sigma-Aldrich (St. Louis, MO, USA), acridine was purchased from Cayman Chemical (Ann Arbor, MI, USA), and lomefloxacin was purchased from Tokyo Chemical Industry Co., Ltd (Tokyo, Japan). Acetone, and sterilized $0.5 \mathrm{w} / \mathrm{v} \%$ methyl cellulose (MC) solution were obtained from Wako Pure Chemical Industries, Ltd. (Osaka, Japan).

\section{Animals}

Male SD rats $[\mathrm{Crl}: \mathrm{CD}(\mathrm{SD})]$ aged 5 weeks were purchased from Charles River Laboratories Japan, Inc. (Kanagawa, Japan). During the acclimation period, clinical observations and measurement of body weight were conducted as part of the quarantine procedures. Clinical signs were checked once a day every day, and body weight was monitored upon arrival and twice per week, using an electronic balance (FX-3000; A\&D Co., Ltd., Tokyo, Japan).

The animals were housed in an animal room, at a temperature maintained between 20 and $26^{\circ} \mathrm{C}$. The humidity was set at $30 \%$ to $70 \%$, air ventilation was set at approximately 17 changes per hr, and $12 \mathrm{hr}$ of light illumination was provided per day (07:00 to 19:00). The animals were placed in suspended rat cages $(\mathrm{W} \times \mathrm{H} \times \mathrm{D}: 260 \times 380$ $\times 180 \mathrm{~mm}$, Model MRS-10; Okazaki Sangyo Co., Ltd., Saitama, Japan). The animals were given free access to pelleted feed for rats (Labo G Standard, Nosan Corporation, Kanagawa, Japan), using attached containers. The animals were allowed free access to tap water (Fujieda City Water) via an automatic water-supply system.

In this study, the animals were handled with care, according to the animal care guidelines of Kaken Pharmaceutical Co., Ltd. (Shizuoka, Japan).

\section{Experimental design}

Experimental design is shown in Fig. 1. For each phototoxic compound, $15 \mathrm{SD}$ rats were selected and divided into three groups: single-dose $(\mathrm{n}=5)$, repeated-dose $(\mathrm{n}=5)$, and repeated-dose plus toxicokinetics (TK; $\mathrm{n}=5$ ). The single-dose group was irradiated with UV-A and UV-B after a single administration of the drug. The repeated-dose and TK groups were irradiated after 8 days of repeated administration of the drug. In addition, blood samples were collected from the TK group on days 1 and 7. 
A general toxicity study incorporating phototoxicity assessments

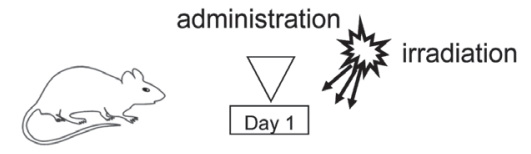

single-dose group
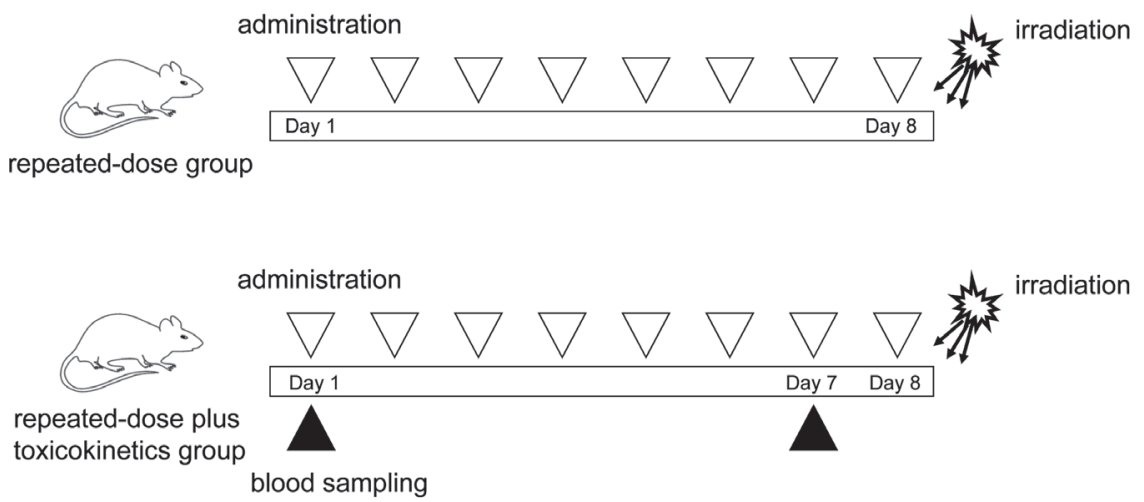

Fig. 1. Experimental design of this study. For each phototoxic compound, 15 SD rats were selected and divided into three groups: single-dose $(n=5)$, repeated-dose $(n=5)$, and repeated-dose plus toxicokinetics (TK; $n=5)$. The single-dose group was irradiated with UV-A and UV-B after a single administration of the drug. The repeated-dose and TK groups were irradiated after 8 days of repeated administration of the drug. In addition, blood samples were collected from the TK group on days 1 and 7.

\section{Phototoxicity assessment protocol of the transdermal administration study}

The phototoxic compounds 8-MOP and acridine were assessed in the transdermal administration study. Acridine and 8-MOP were dissolved in acetone to give a $5 \mathrm{w} / \mathrm{v} \%$ and $10 \mathrm{w} / \mathrm{v} \%$ solution, respectively. The entire backs of the animals were shaved using an electric clipper (6000AD; Daito Electric Machine Industry Co., Ltd., Osaka, Japan) and an electric shaver (LS5500; Braun, Kronberg, Germany). A portion of the test compound solution $(20 \mu \mathrm{L})$ was then applied to two application sites $\left(1.5 \times 1.5 \mathrm{~cm}^{2} / \mathrm{site}\right)$ on the back of the animal. The animals were sedated with pentobarbital ( $50 \mathrm{mg} / \mathrm{kg}$, ip) immediately before irradiation. At $30 \mathrm{~min}$ after application of the test compound, one half of the back of each animal was covered with aluminum foil to make irradiation and nonirradiation sites. Then, the animals were irradiated using a UV irradiation device with UV-A light source (FL20S BL/DMR; Toshiba Medical Systems Corporation, Tokyo, Japan) and UV-B light source (TL20W/12RS; Philips, Amsterdam, Netherlands). A glass filter (3-mm thick) was placed between the UV-A light source and the skin, to block UV light with a wavelength below $320 \mathrm{~nm}$. The UV-A and UV-B light sources were positioned $10 \mathrm{~cm}$ and $30 \mathrm{~cm}$ away from the surface of the skin. The dose of UV-A was set at $10 \mathrm{~J} / \mathrm{cm}^{2}$, as is used in typical skin pho- totoxicity studies (ICH guideline S10, 2013). The dose of UV-B was set at $0.031 \mathrm{~J} / \mathrm{cm}^{2}$, based on a previous report (Yonezawa et al., 2015). Skin reactions (erythema/eschar and edema formation) were observed 24,48 , and $72 \mathrm{hr}$ after UV irradiation, according to the Draize method (Table 1) (Draize, 1959). Skin phototoxicity was evaluated by comparing the skin reactions of the irradiation and non-irradiation sites.

\section{Phototoxicity assessment protocol of the oral administration study}

The phototoxic compounds 8-MOP and lomefloxacin were assessed in the oral administration study. Lomefloxacin and 8-MOP were dissolved in sterilized $0.5 \mathrm{w} / \mathrm{v} \% \mathrm{MC}$, and administered at doses of $10 \mathrm{mg} / \mathrm{kg}$ and $80 \mathrm{mg} / \mathrm{kg}$, respectively. The entire backs of the animals were shaved using an electric clipper and an electric shaver. The test compound was orally administered to the animals once a day, at a dosing volume of $10 \mathrm{~mL} / \mathrm{kg}$. The animals were then sedated with pentobarbital $(50 \mathrm{mg} / \mathrm{kg}$, ip) immediately before irradiation. One half of the back of each animal was covered with aluminum foil, and the animals were irradiated as for the transdermal administration study. The irradiation start time was set based on a previous report (Yonezawa et al., 2015), to ensure that irradiation occurred at around $\mathrm{T}_{\max }$. The time- 
Table 1. Evaluation of Skin Reactions (Draize's Criteria: 1959).

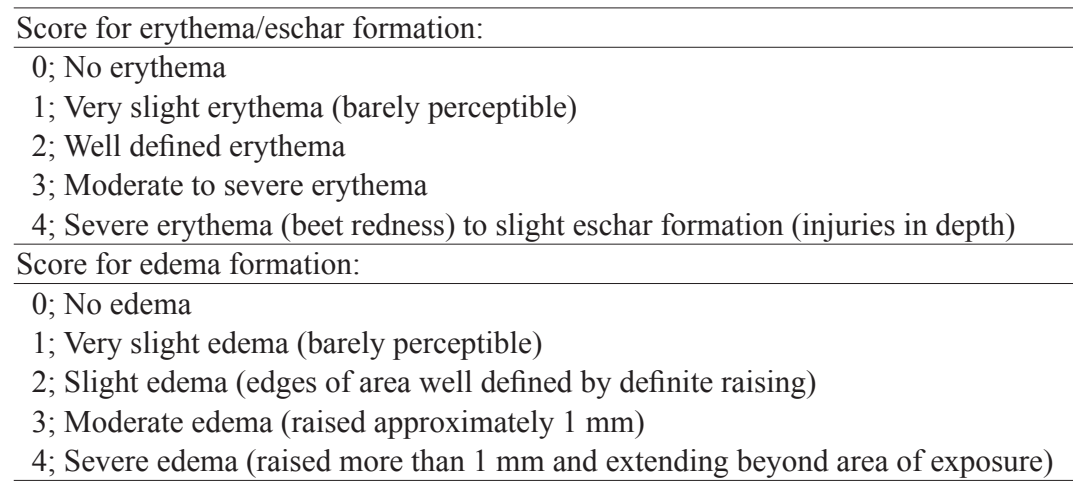

points used were $0.5 \mathrm{hr}$ for 8 -MOP and $1.0 \mathrm{hr}$ for lomefloxacin. Skin reactions were observed as for the transdermal administration study.

\section{Skin reaction evaluation}

For each test group, the skin reaction scores (erythema/ eschar and edema formation) of individual animals were summed for each site, and the mean score was calculated according to the following equation:

Mean score $=$ Total of erythema and edema scores / Number of animals tested.

The test compound was judged to be phototoxic if the mean score of the UV-irradiated site was higher than that of the non-irradiated site at any observation period.

Additionally, the total of the erythema and edema scores for the UV-irradiated site was analyzed using Bartlett's test for homogeneity of variance. When the variance was homogeneous, a Tukey test for multiple comparisons was performed between each of the test groups. When the variance was heterogeneous, a Steel-Dwass test for multiple comparisons was performed between each of the test groups.

Statistical analysis software (EXSUS Version 8.1, CAC Croit Corporation, Tokyo, Japan) was used to calculate the mean and standard deviation in each group. A two-sided significance level of 5\% was used for the statistical analyses.

\section{Blood sampling for the TK analysis}

Blood samples were collected from the TK group $(n=5)$ on days 1 and 7 of administration. Blood was collected at six time points in total: before dosing, and 0.5 , $1,4,8$ and $24 \mathrm{hr}$ after dosing. Approximately $300 \mu \mathrm{L}$ of blood was collected from the caudal vein of each animal, using a micropipette with a disposable tip. The blood was then immediately transferred to a $0.5-\mathrm{mL}$ polypropylene tube containing $6 \mu \mathrm{L}$ of heparin sodium
(Novo-Heparin 5,000 units/5 mL for injection; Mochida Pharmaceutical Co., Ltd., Tokyo, Japan) and mixed. The samples were then kept under ice-cold conditions until use. Plasma samples were obtained by centrifugation $\left(4^{\circ} \mathrm{C}, 3,000 \mathrm{rpm}, 15 \mathrm{~min}\right)$, using a refrigerated centrifuge (05PR-22; Hitachi Koki Co., Ltd., Tokyo, Japan).

\section{Measurement of drug concentration Plasma extraction procedure for 8-MOP and acridine}

For plasma extraction of 8-MOP and acridine, 25 $\mu \mathrm{L}$ of the plasma sample, $5 \mu \mathrm{L}$ of internal standard (IS) solution $(1,000 \mathrm{ng} / \mathrm{mL}$ trioxsalen acetonitrile solution), $200 \mu \mathrm{L}$ of $2 \%$ formic acid (v/v) in water, $25 \mu \mathrm{L}$ of acetonitrile and $2 \mathrm{~mL}$ of t-butyl methyl ether were pipetted into a glass tube, and centrifuged at $3,000 \times \mathrm{g}$ at $4^{\circ} \mathrm{C}$ for $10 \mathrm{~min}$. The supernatant of the t-butyl methyl ether layer was then transferred to a new glass tube, and dried under reduced pressure. The residue was reconstituted with 50 $\mu \mathrm{L}$ of dissolving solution ( $50 \%$ acetonitrile solution (v/v)) and added to a microreaction tube prior to injection into the analytical system.

For the calibration standard and quality control (QC) samples, $25 \mu \mathrm{L}$ of blank rat plasma, $5 \mu \mathrm{L}$ of IS solution, $25 \mu \mathrm{L}$ of working standard solution $(25 \mu \mathrm{L}$ of acetonitrile for the zero sample), and $2 \mathrm{~mL}$ of t-butyl methyl ether were pipetted into a glass tube, and then the same procedures as mentioned above were performed.

\section{Plasma extraction procedure for lomefloxacin}

For plasma extraction of lomefloxacin, $25 \mu \mathrm{L}$ of the plasma sample, $25 \mu \mathrm{L}$ of IS solution $(500 \mathrm{ng} / \mathrm{mL}$ ketoconazole methanol solution), and $50 \mu \mathrm{L}$ of methanol were pipetted into a polypropylene tube, and centrifuged at $21,600 \times \mathrm{g}$ at $4^{\circ} \mathrm{C}$ for $10 \mathrm{~min}$. The supernatant was then added into a microreaction tube prior to injection into the analytical system. 
A general toxicity study incorporating phototoxicity assessments

For the calibration standard and QC samples, $25 \mu \mathrm{L}$ of blank rat plasma, $25 \mu \mathrm{L}$ of IS solution, $25 \mu \mathrm{L}$ of working standard solution $(25 \mu \mathrm{L}$ of methanol for the zero sample), and $25 \mu \mathrm{L}$ of methanol were pipetted into a polypropylene tube, and then the same procedures as mentioned above were performed.

\section{LC-MS/MS conditions}

After extraction, the plasma concentrations of the drugs were analyzed by liquid chromatography-mass spectrometry (LC-MS/MS). The LC system consisted of a pump, a Nanospace SI-2 autosampler (Shiseido, Tokyo, Japan) and a C18 reversed-phase analytical column (Ascentis Express C18, $2.1 \mathrm{~mm}$ I.D. x $30 \mathrm{~mm}, 2.7 \mu \mathrm{m}$; SUPELCO, Bellefonte, PA, USA), and was connected to a TSQ Quantum Ultra Triple-Quadrupole mass spectrometer (Thermo Fisher Scientific, Waltham, MA, USA). The injection volume was $10 \mu \mathrm{L}$. Gradient elution was performed with mobile phase $\mathrm{A}$, which consisted of $0.1 \%$ formic acid (v/v) in water (98\%), and mobile phase B, which consisted of $0.1 \%$ formic acid (v/v) in methanol $(2 \%)$. The following gradient program was used at a flow rate of $0.4 \mathrm{~mL} / \mathrm{min}$ : $0-0.1 \mathrm{~min}$, linear gradient from $98 \%$ A to $0 \% \mathrm{~A}(\mathrm{v} / \mathrm{v}) ; 0.1-1.6 \mathrm{~min}$, hold at $0 \% \mathrm{~A} ; 1.6-1.7 \mathrm{~min}$, linear gradient from $0 \%$ A to $98 \% \mathrm{~A}(\mathrm{v} / \mathrm{v}) ; 1.7-4.2 \mathrm{~min}$, hold at $98 \% \mathrm{~A}$. Quantification of the protonated precursor ion and the related product ion was performed in MRM mode using an internal standard method with peak area ratios. The mass transitions used for quantification were $\mathrm{m} / \mathrm{z}$ $217.0 \rightarrow 174.0, \mathrm{~m} / \mathrm{z} 180.0 \rightarrow 152.0, \mathrm{~m} / \mathrm{z} 229.1 \rightarrow 141.0, \mathrm{~m} / \mathrm{z}$ $352.1 \rightarrow 236.9$ and $\mathrm{m} / \mathrm{z} 531.3 \rightarrow 243.9$, for 8 -MOP, acridine, trioxsalen, lomefloxacin and ketoconazole, respectively. The quantitative range of the calibration curve was $1 \sim 100 \mathrm{ng} / \mathrm{mL}, 1 \sim 200 \mathrm{ng} / \mathrm{mL}$ and $0.1 \sim 10 \mu \mathrm{g} / \mathrm{mL}$, for 8-MOP, acridine and lomefloxacin, respectively.

\section{Data analysis for plasma concentration}

XcaliburTM and LCquanTM software (Thermo Fisher Scientific) was used for data acquisition and analysis. The linearity of the calibration curves was assessed using weighted $(1 / y)$ least-squares linear regression of the analyte : IS peak area ratio. The mean and standard deviation of TK parameters were calculated with Excel 2010 (Microsoft Corporation, Redmond, WA, USA).

\section{RESULTS}

\section{Skin phototoxicity assessment in the transdermal administration study}

The skin scores obtained in the transdermal administration study are shown in Fig. 2. As representative examples, images showing the skin reactions caused by 8-MOP and acridine are shown in Figs. 3 and 4, respectively.

Among the 8-MOP-treated groups, the single-dose

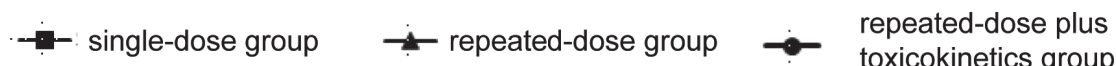

\section{A}

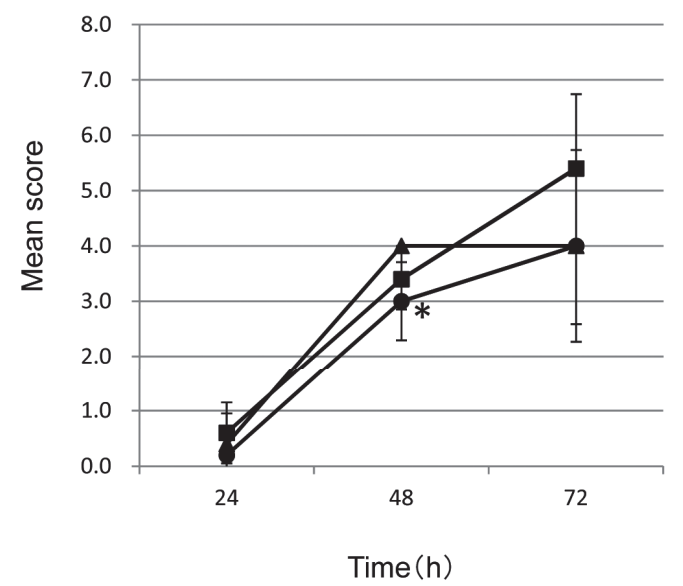

B

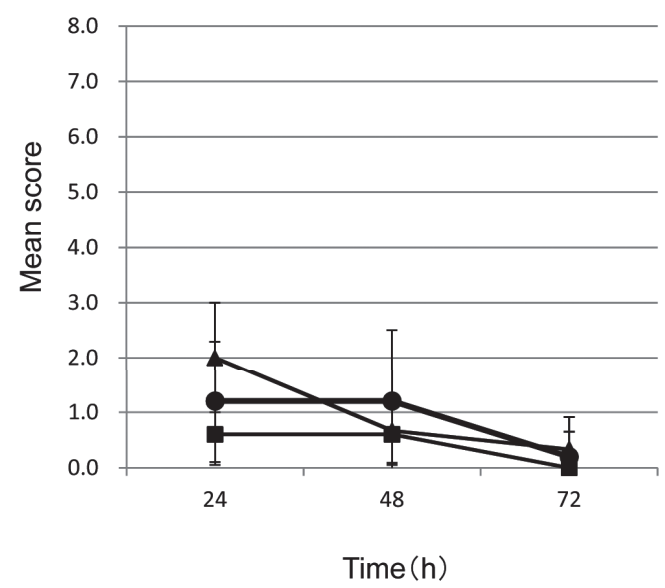

Fig. 2. Plot of the skin scores of the UV irradiated site for the compounds tested in the transdermal administration study: (A) 8-MOP and (B) acridine. The skin score of the repeated-dose plus toxicokinetics group treated with 8-MOP was significantly different from that of the repeated-dose group, ${ }^{*} \mathrm{P}<0.05$ (Tukey test for multiple comparisons). Each point represents the mean $\pm \mathrm{SD}$ values of five animals, except for the repeated-dose group treated with acridine $(\mathrm{n}=3)$. The data for the nonirradiated site are not shown, because the scores were 0 for both compounds. 
Y. Yonezawa et al.

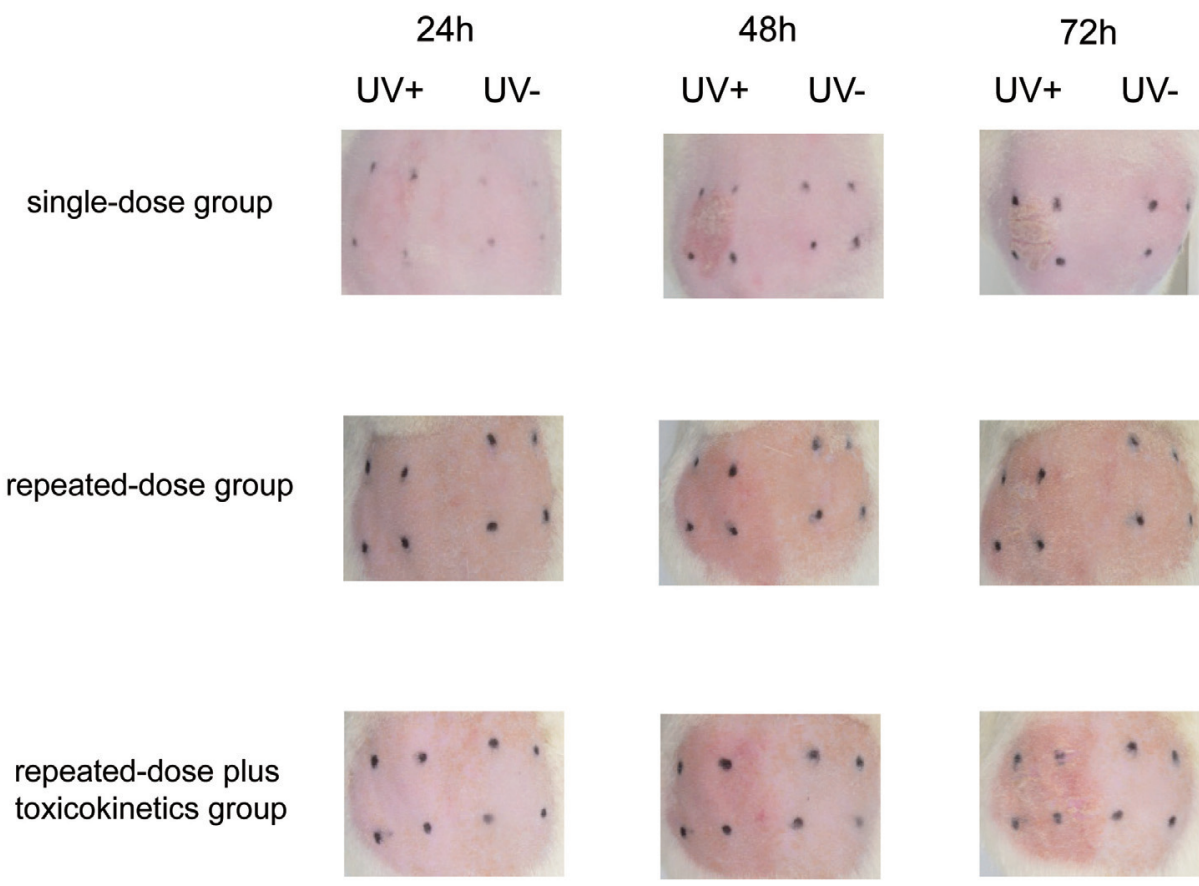

Fig. 3. Image showing the skin of an SD rat treated with 8-MOP in the transdermal administration study.

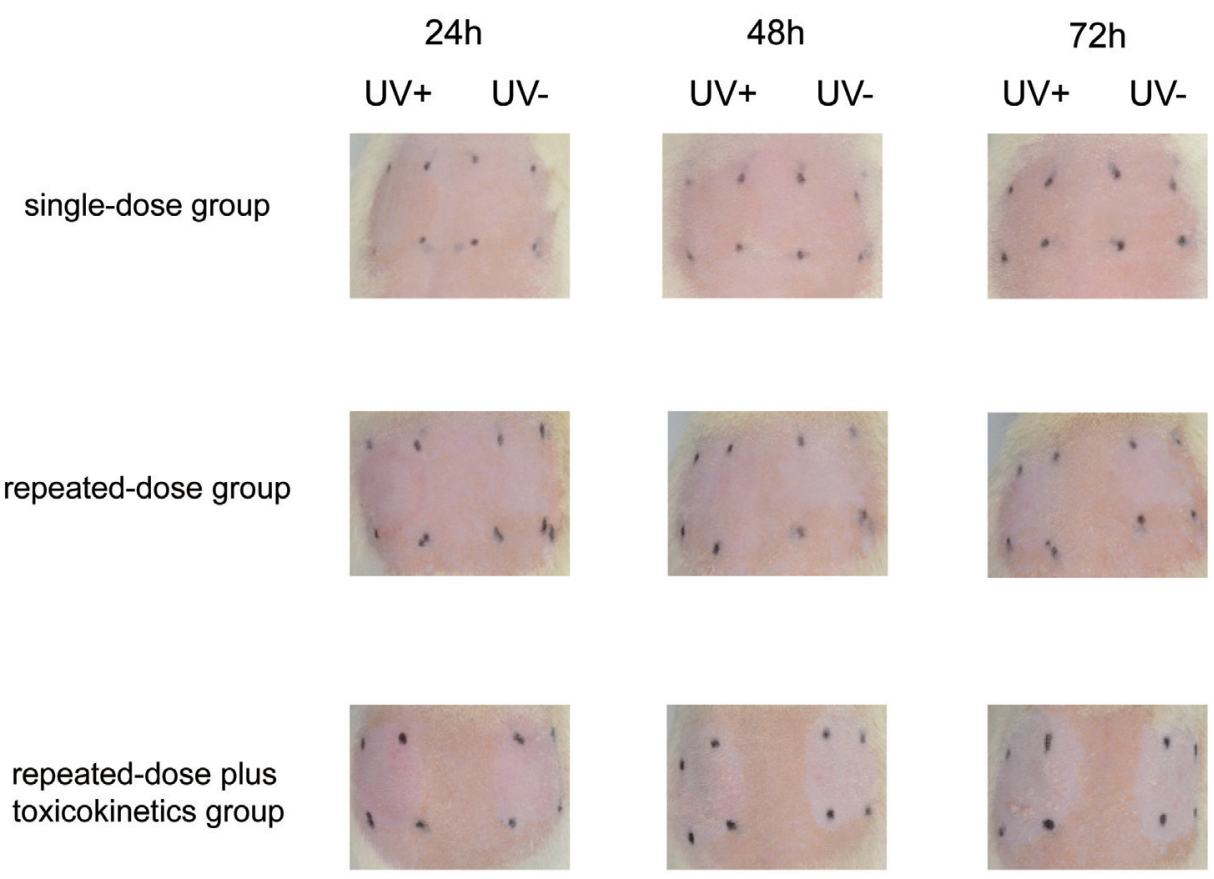

Fig. 4. Image showing the skin of an SD rat treated with acridine in the transdermal administration study. 
A general toxicity study incorporating phototoxicity assessments

group showed evidence of slight phototoxicity, such as very slight erythema, at $24 \mathrm{hr}$ after irradiation. However, the phototoxic damage gradually increased up to severe erythema and slight edema $72 \mathrm{hr}$ after irradiation. The repeated-dose and TK groups showed evidence of mild phototoxicity, such as well-defined erythema, $24 \mathrm{hr}$ after irradiation. However, over time, the phototoxic damage gradually increased up to the same degree as that seen in the single-dose group.

Among the acridine-treated groups, the single-dose group similarly showed slight evidence of phototoxicity, including very slight erythema, $24 \mathrm{hr}$ after irradiation. However, the signs of phototoxicity gradually decreased over time, and the skin reaction had disappeared $72 \mathrm{hr}$ after irradiation. The repeated-dose and TK groups also showed mild phototoxic damage, including very slight erythema and edema, $24 \mathrm{hr}$ after irradiation. Similar to that in the single-dose group, the phototoxic damage gradually decreased over time. Two of the animals in the repeated-dose group could not be irradiated properly, as one of them died because of an overdose of the anesthetic and the other escaped from the irradiated area. Thus, these animals were excluded from the evaluation.

In both the 8-MOP- and acridine-treated groups, there was no skin reaction at the non-irradiated site. Thus, 8-MOP and acridine were accurately judged to be phototoxic in all of the groups. Additionally, the total of the erythema and edema scores of the UV-irradiated site significantly decreased in the TK group treated with 8-MOP, compared with that in the repeated-dose group.

\section{Skin phototoxicity assessment in the oral administration study}

The skin scores obtained in the oral administration study are shown in Fig. 5. As representative examples, images showing the skin reactions caused by 8-MOP and lomefloxacin are shown in Figs. 6 and 7, respectively.

Among the 8-MOP-treated groups, the single-dose group showed evidence of slight phototoxicity, such as very slight erythema and edema, $24 \mathrm{hr}$ after irradiation. The phototoxic skin reaction then gradually worsened, resulting in severe erythema and edema $72 \mathrm{hr}$ after irradiation. The repeated-dose and TK groups showed slight phototoxic damage, including well-defined erythema but no edema, $24 \mathrm{hr}$ after irradiation. The phototoxic damage then gradually increased up to severe erythema and mild edema $72 \mathrm{hr}$ after irradiation.

Among the lomefloxacin-treated groups, the singledose group showed mild phototoxic damage, such as welldefined erythema and very slight edema, $24 \mathrm{hr}$ after irradiation. However, the signs of phototoxicity then gradually decreased down to well-defined erythema $72 \mathrm{hr}$ after irradiation. The repeated-dose and TK groups also showed mild phototoxic damage, such as well-defined erythema,

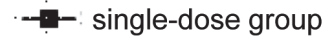

A

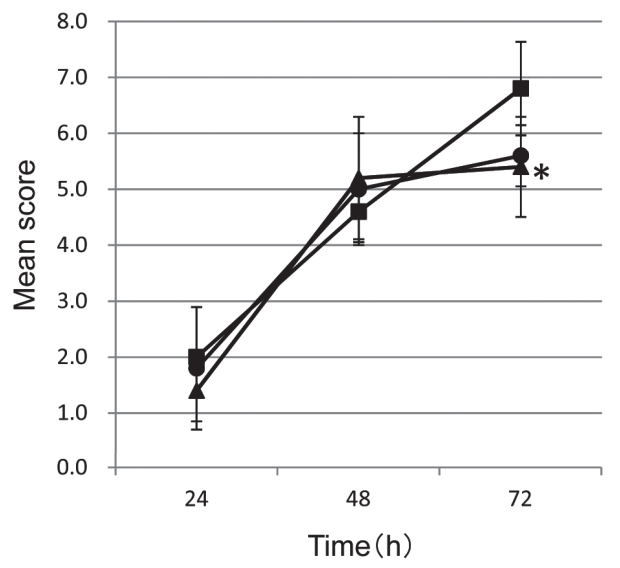

repeated-dose plus repeated-dose group $\longrightarrow \begin{aligned} & \text { repeated-dose plus } \\ & \text { toxicokinetics group }\end{aligned}$

Fig. 5. Plot of the skin scores of the UV-irradiated site for the compounds tested in the oral administration study: (A) 8-MOP and (B) lomefloxacin. The skin score of the repeated-dose group treated with 8-MOP was significantly different from that of the single-dose group, $* \mathrm{P}<0.05$ (Tukey test for multiple comparisons). Each point represents the mean \pm S.D. values of five animals. The data for the non-irradiated site are not shown, because the scores were 0 for both compounds. 
Y. Yonezawa et al.

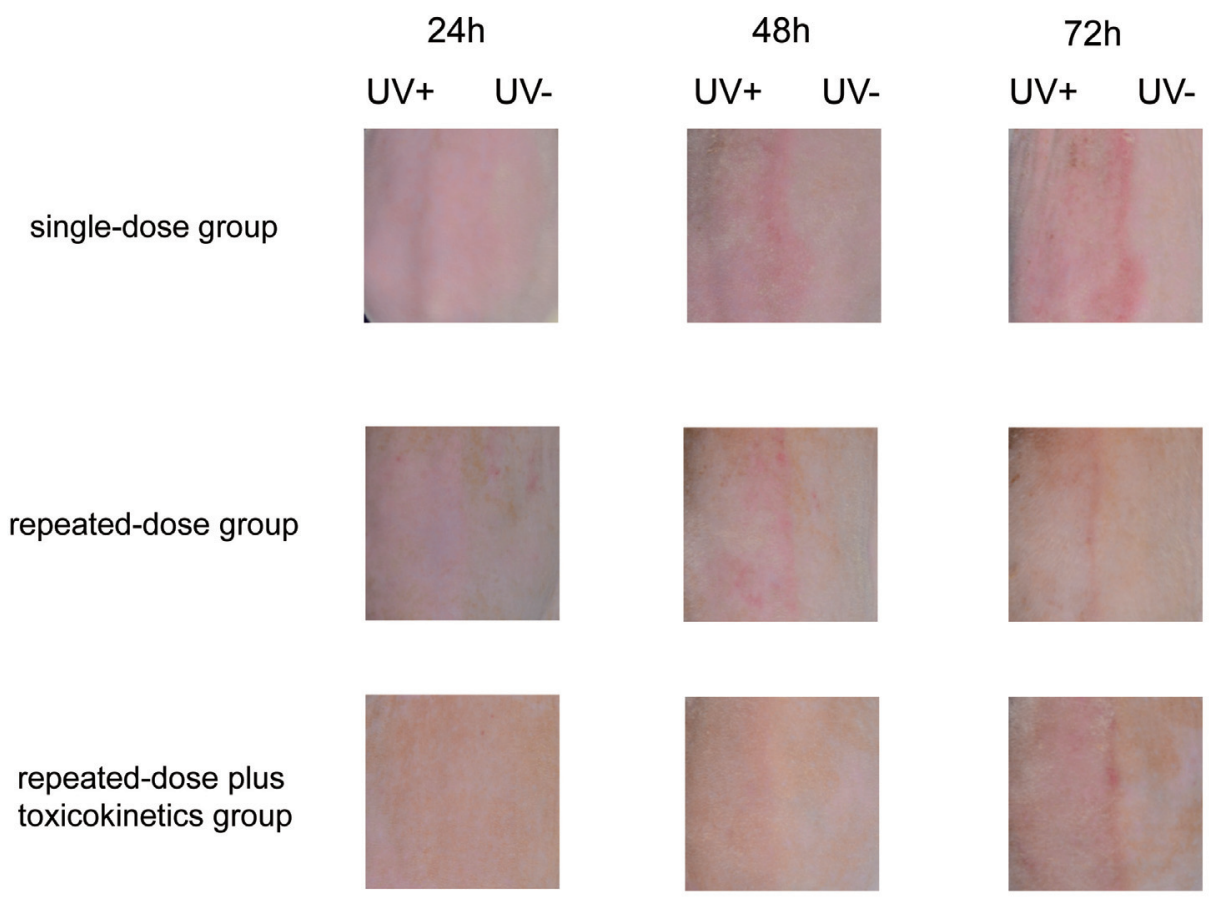

Fig. 6. Image showing the skin of an SD rat treated with 8-MOP in the oral administration study.

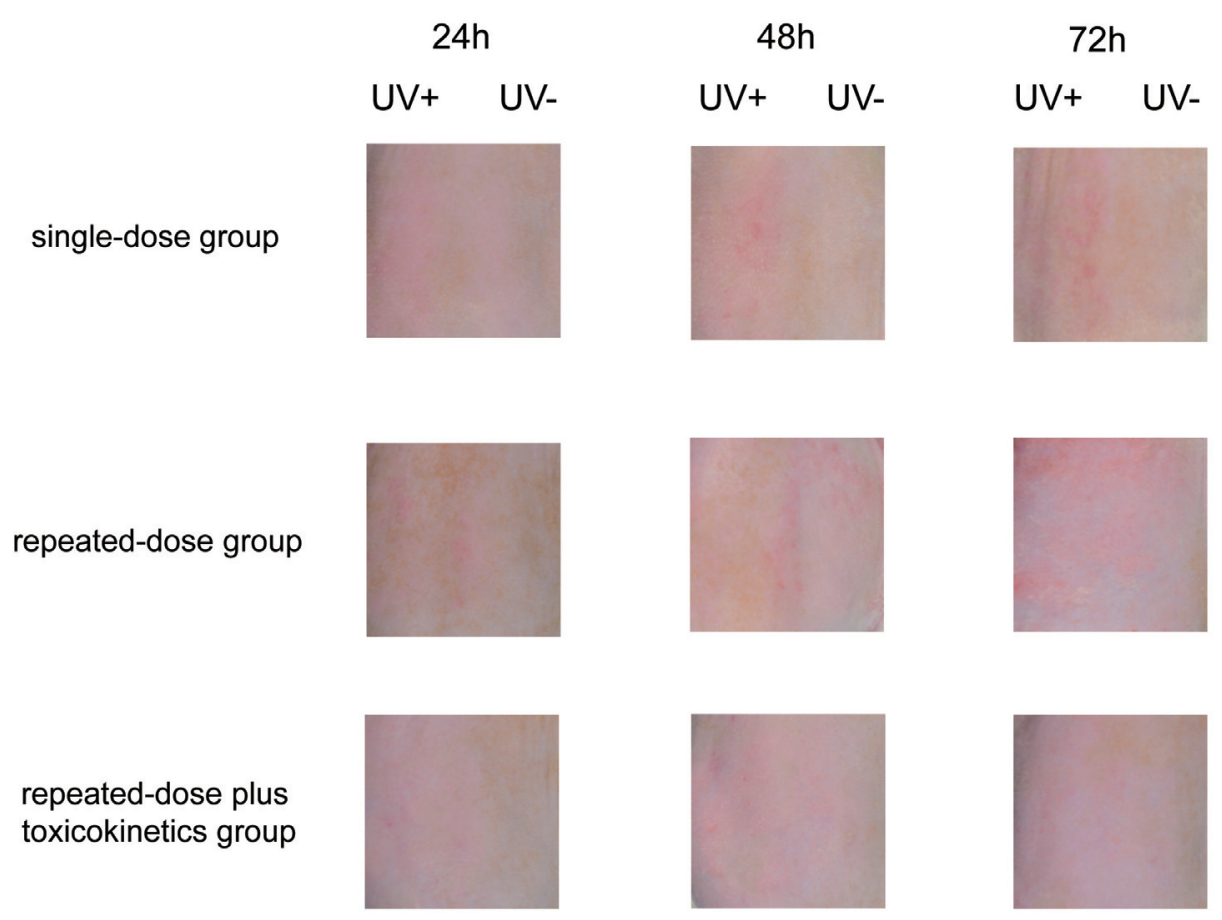

Fig. 7. Image showing the skin of an SD rat treated with lomefloxacin in the oral administration study. 
A general toxicity study incorporating phototoxicity assessments

$24 \mathrm{hr}$ after irradiation. Similar to that in the single-dose group, the signs of phototoxicity gradually decreased over time, but the reaction $72 \mathrm{hr}$ after irradiation was slightly weaker than that seen in the single-dose group.

In both the 8-MOP- and lomefloxacin-treated groups, there was no skin reaction at the non-irradiated site. Thus, 8-MOP and lomefloxacin were accurately judged to be phototoxic in all of the groups. Additionally, the total of the erythema and edema scores of the UV-irradiated site significantly decreased in the repeated-dose group treated with 8-MOP, compared with that in the single-dose group.

\section{Toxicokinetics}

The TK parameters of each compound are summarized in Tables 2 and 3. The concentrations of each compound in the plasma after transdermal or oral administration are summarized in Figs. 8 and 9.

In the transdermal administration study, repeated administration decreased the maximum concentration $\left(\mathrm{C}_{\max }\right)$ and area under the curve $\left(\mathrm{AUC}_{0-24}\right)$ values in the 8-MOP-treated group. In the acridine-treated group, there were no clear changes in the $\mathrm{C}_{\max }$ or $\mathrm{AUC}_{0-24}$ values after repeated dosing. The $\mathrm{T}_{\max }$ values of 8 -MOP and acridine were $2.3 \mathrm{hr}$ and $4.4 \mathrm{hr}$ after administration, respective-

Table 2. Summary of TK parameters in transdermal administration study.

Day1

\begin{tabular}{lccccc}
\hline & Units & $5 \mathrm{w} / \mathrm{v} \% 8-\mathrm{MOP}$ & $\mathrm{N}$ & $10 \mathrm{w} / \mathrm{v} \%$ Acridin & $\mathrm{N}$ \\
\hline $\mathrm{C}_{\max }$ & $\mathrm{ng} / \mathrm{mL}$ & $148 \pm 215$ & 5 & $54.6 \pm 24.0$ & 5 \\
$\mathrm{~T}_{\max }$ & $\mathrm{hr}$ & $2.3 \pm 3.2$ & 5 & $4.4 \pm 3.5$ & 5 \\
$\mathrm{~T}_{1 / 2}$ a) & $\mathrm{hr}$ & 111 & 2 & 22.0 & 2 \\
$\mathrm{AUC}_{0-24}$ & $\mathrm{ng} \cdot \mathrm{hr} / \mathrm{mL}$ & $838 \pm 1119$ & 5 & $751 \pm 287$ & 5 \\
\hline
\end{tabular}

(mean \pm S.D.)

Day7

\begin{tabular}{lccccc}
\hline & Units & $5 \mathrm{w} / \mathrm{v} \% 8-\mathrm{MOP}$ & $\mathrm{N}$ & $10 \mathrm{w} / \mathrm{v} \%$ Acridin & $\mathrm{N}$ \\
\hline $\mathrm{C}_{\max }$ & $\mathrm{ng} / \mathrm{mL}$ & $17.3 \pm 9.9$ & 5 & $90.8 \pm 63.5$ & 5 \\
$\mathrm{~T}_{\max }$ & $\mathrm{hr}$ & $1.1 \pm 1.6$ & 5 & $3.4 \pm 3.1$ & 5 \\
$\mathrm{~T}_{1 / 2}{ }^{a}$ & $\mathrm{hr}$ & $62.8 \pm 19.2$ & 3 & 23.6 & 2 \\
$\mathrm{AUC}_{0-24}$ & $\mathrm{ng} \cdot \mathrm{hr} / \mathrm{mL}$ & $129 \pm 37$ & 5 & $832 \pm 609$ & 5 \\
\hline
\end{tabular}

(mean \pm S.D.)

a): In order to calculate $T_{1 / 2}$, the plasma concentrations of the drugs must be detected at three or more time points after $T_{\max }$. However, this was not possible for some animals, and these animals were excluded from the assessment.

Table 3. Summary of TK parameters in oral administration study.

Day1

\begin{tabular}{lccccc}
\hline & Units & $1 \mathrm{mg} / \mathrm{mL} 8-\mathrm{MOP}$ & $\mathrm{N}$ & $8 \mathrm{mg} / \mathrm{mL}$ Lomefloxacin & $\mathrm{N}$ \\
\hline $\mathrm{C}_{\max }$ & $\mathrm{ng} / \mathrm{mL}$ & $3212 \pm 789$ & 5 & $7520 \pm 1423$ & 5 \\
$\mathrm{~T}_{\max }$ & $\mathrm{hr}$ & $1.0 \pm 0.0$ & 5 & $0.8 \pm 0.3$ & 5 \\
$\mathrm{~T}_{1 / 2}$ a) & $\mathrm{hr}$ & $\mathrm{NC}$ & $\mathrm{NC}$ & $2.3 \pm-$ & 2 \\
$\mathrm{AUC}_{0-24}$ & $\mathrm{ng} \cdot \mathrm{hr} / \mathrm{mL}$ & $12107 \pm 3484$ & 5 & $36817 \pm 3014$ & 5 \\
\hline
\end{tabular}

(mean \pm S.D.)

Day7

\begin{tabular}{lccccc}
\hline & Units & $1 \mathrm{mg} / \mathrm{mL} 8-\mathrm{MOP}$ & $\mathrm{N}$ & $8 \mathrm{mg} / \mathrm{mL}$ Lomefloxacin & $\mathrm{N}$ \\
\hline $\mathrm{C}_{\max }$ & $\mathrm{ng} / \mathrm{mL}$ & $2206 \pm 471$ & 5 & $6770 \pm 959$ & 5 \\
$\mathrm{~T}_{\max }$ & $\mathrm{hr}$ & $1.0 \pm 0.0$ & 5 & $0.9 \pm 0.2$ & 5 \\
$\mathrm{~T}_{1 / 2}^{\text {a) }}$ & $\mathrm{hr}$ & $\mathrm{NC}$ & $\mathrm{NC}$ & $\mathrm{NC}$ & $\mathrm{NC}$ \\
$\mathrm{AUC}_{0-24}$ & $\mathrm{ng} \cdot \mathrm{hr} / \mathrm{mL}$ & $7041 \pm 1467$ & 5 & $35244 \pm 958$ & 5 \\
\hline
\end{tabular}

(mean \pm S.D.)

NC: Not calculated

a): In order to calculate $T_{1 / 2}$, the plasma concentrations of the drugs must be detected at three or more time points after $T_{\max }$. However, this was not possible for some or all animals, and these animals were excluded from the assessment. 


\section{Y. Yonezawa et al.}
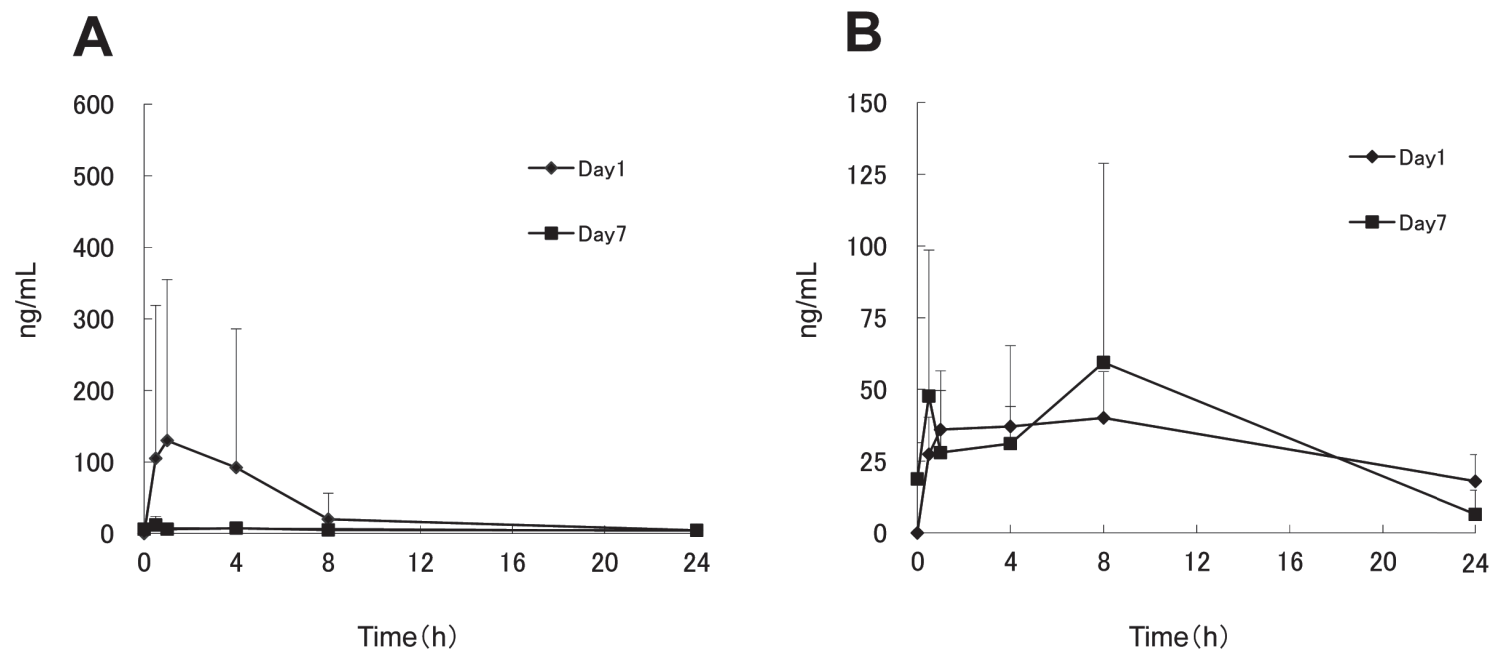

Fig. 8. Plasma drug concentrations after transdermal administration to SD rats. (A) 8-MOP, (B) acridine. Each point represents the mean \pm S.D. of five animals.
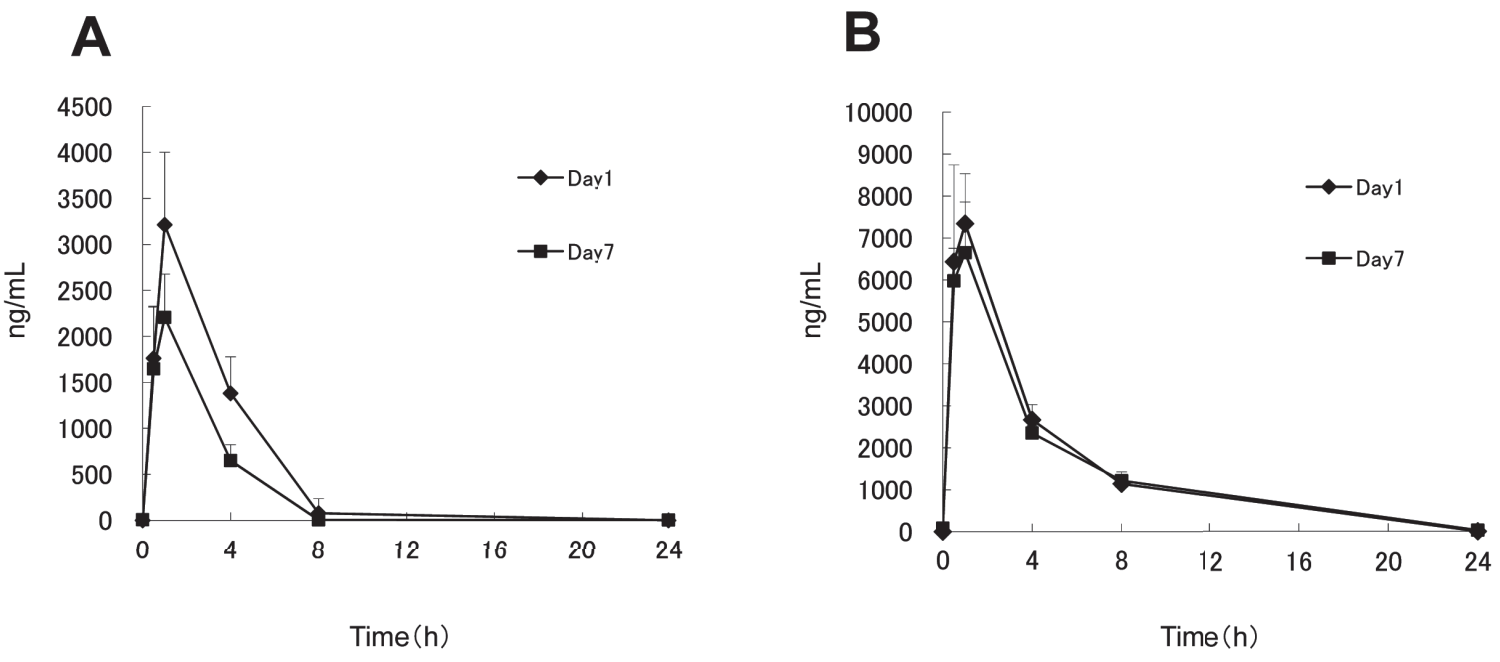

Fig. 9. Plasma drug concentrations after oral administration to SD rats. (A) 8-MOP, (B) lomefloxacin. Each point represents the mean \pm S.D. of five animals.

1y. In both groups, the plasma concentration of the compound was near to the $\mathrm{C}_{\max } 0.5 \mathrm{hr}$ after administration, the time of UV irradiation, although the $\mathrm{T}_{\max }$ values varied widely.

In the oral administration study, there were no clear changes in the $\mathrm{C}_{\max }$ or $\mathrm{AUC}_{0-24}$ values after repeated dosing in the 8-MOP and lomefloxacin groups. The $\mathrm{T}_{\max }$ values of 8-MOP and lomefloxacin were $1.0 \mathrm{hr}$ and $0.8 \mathrm{hr}$ after administration, respectively. In both groups, the plasma concentration of the compound was near to the $C_{\max } 0.5 \mathrm{hr}$ after administration, the time of UV irradiation.

\section{DISCUSSION}

Incorporating various assessments into general toxicity studies is recommended by the ICH Guideline S2(R1), 2008, ICH Guideline M3(R2), 2009 and FDA Guidance for Industry Photosafety Testing, 2003. However, there are two potential obstacles to performing such studies. The first is the effect of the general toxicity study on addi- 
A general toxicity study incorporating phototoxicity assessments

tional assessments. General toxicity studies may affect additional assessments because they require additional administration, sampling, and observation, which are not normally needed in additional assessments, and may have an impact on the results of additional assessments. The other potential obstacle is the effect of additional assessments on the general toxicity study, as additional assessments similarly require additional administration, sampling, and observation, which are not needed in a normal general toxicity study. For example, to perform a comet assay, it is necessary to autopsy the animal 2-6 hr after the final administration (Tice et al., 2000). This does not fit the study schedule of a general toxicity study, and so incorporation of a comet assay into a general toxicity study would be very difficult.

The present study aimed to examine whether skin phototoxicity assessments could be incorporated into general toxicology studies, using SD rats. The results showed that, regardless of the route of administration, the singledose, repeated-dose, and TK groups all showed phototoxic reactions.

In the transdermal administration study, the skin score of the UV-irradiated site in the TK group treated with 8-MOP significantly decreased when compared with that in the repeated-dose group. However, this reaction was observed at only one time-point (48 $\mathrm{hr}$ after irradiation), and there was no significant change when compared with that in the single-dose group. Thus, the statistical significance of this reaction was considered due to the low value of standard deviation in the repeated-dose group (S.D. was 0.0 ) and therefore this reaction was considered not to be treatment-related. In this study, one rat died because of an overdose of anesthetic. We determined that the reason for this overdose was that a high concentration of pentobarbital was absorbed due to a vascular injury that occurred during intraperitoneal administration of the treatment. Furthermore, this death was the only one caused by an overdose of anesthetic in the transdermal and oral administration study, which included 60 animals. Therefore, we concluded that most of the animals were sedated with an appropriate amount of pentobarbital. Regarding the effect of anesthesia on the pharmacokinetics, there should be almost no effect because the animals were sedated immediately before irradiation, whereas the drug was administered $0.5 \mathrm{hr}$ before irradiation. Therefore, the drug should have distributed in the skin sufficiently before anesthesia occurred. Moreover, in a previous report (Yonezawa et al., 2015), we showed that the phototoxicity of multiple drugs can be detected under the same anesthesia conditions. Thus, it was confirmed that pentobarbital anesthesia did not affect the phototoxicity evaluation.
In the oral administration study, the UV-irradiated skin score of the repeated-dose group treated with 8-MOP significantly decreased when compared with that in the single-dose group. However, this reaction was also observed at only one time-point ( $72 \mathrm{hr}$ after irradiation), and there was no significant change between the single-dose group and TK group, which was treated equally as or more severely than the repeated-dose group. Therefore, this reaction was also considered not to be treatment-related. Thus, it was confirmed that there was no significant change between the single-dose group and repeated-dose group. This result corresponded with the result from the previous study using Long-Evans rats (Morimura et al., 2010). This study evaluated the phototoxicity of 8-MOP and lomefloxacin with single-dose group and 7-day repeated-dose group, and concluded that there was no difference in the skin reaction of the albino area between both groups. Additionally, it was confirmed that blood collection for the TK study did not affect the skin phototoxicity assessment, and the phototoxic potential could be evaluated in the TK group. This proves that the first obstacle given above does not matter in this study.

In addition, the TK measurements helped successfully detect all of the phototoxic compounds in the plasma samples, and it was confirmed that the UV irradiation timing was close to $\mathrm{T}_{\max }$. No major differences in the $\mathrm{T}_{\max }$, $\mathrm{C}_{\max }$, or $\mathrm{AUC}_{0-24}$ values were observed throughout the dosing period, except in the 8-MOP-treated group in the transdermal administration study. In this group, repeated administration decreased the $\mathrm{C}_{\max }$ and $\mathrm{AUC}_{0-24}$ values. However, the reason for this decrease was not considered to be enzyme induction, because very high exposure (the $\mathrm{C}_{\max }$ value was $530 \mathrm{ng} / \mathrm{mL}$, and the $\mathrm{AUC}_{0-24}$ value was $2,755 \mathrm{ng} \cdot \mathrm{hr} / \mathrm{mL}$ ) was observed in one animal on Day 1 (Table 2), and the score of this animal was considered to increase the $\mathrm{C}_{\max }$ and $\mathrm{AUC}_{0-24}$ values on Day 1 . The reason for the absorption being increased in this animal may be a scratch on the skin caused by the shaving procedure (Banerjee and Ritschel, 1989) or oral intake, but the details are currently unclear and further investigations are needed.

Regarding the second obstacle highlighted above, when incorporating a phototoxicity assessment into a general toxicity study, the phototoxicity assessment should have no effect on the general toxicity study, because the phototoxicity assessment in the animals occurs after TK blood collection is complete.

Thus, the results of the present study confirm that there are no obstacles to incorporating phototoxicity assessments. However, further studies are needed in order to confirm that this toxicity evaluation method can also 


\section{Y. Yonezawa et al.}

be used to evaluate drugs with accumulative properties, like meloxicam (Seto et al., 2013; Türck et al., 1997), or that are degraded by metabolic enzymes, like amiodarones (Allen, 1993; Elsherbiny et al., 2008; Yonezawa et al., 2015). These drugs may affect the TK parameters throughout the dosing period, and may cause different phototoxic reactions between the first administration and final administration.

Although such further studies are needed, there are a number of potential advantages to being able to incorporate phototoxicity assessments and general toxicity evaluations into a single study. For example, in this study, the plasma concentration data from the TK group was not used to determine the irradiation timing, because the irradiation time had already been determined in advance. However, these results show that there is no need to obtain the plasma drug concentration data before the phototoxicity assessment. Instead, both assessments can be performed at the same time, in the same animal, which will help to speed up phototoxic evaluations. Additionally, in the general toxicity study, multiple dosages are evaluated using both genders, while a single dosage is evaluated using one gender in the phototoxicity study. Thus, differences between the sexes and dose-response can also be evaluated with this toxicity evaluation method. This meets the ICH guideline S10 (2013) statement that "If a negative result is obtained at the maximum dose, testing of lower doses is usually not warranted. However, if a positive result is anticipated, additional dose groups can support a NOAEL-based risk assessment."

Moreover, incorporating phototoxicity assessments reduces the number of studies and the number of animals required. This contributes to shortening the research and development period, and helps support the 3 Rs principle for animal experiments.

Finally, incorporating phototoxicity assessments means that TK data, which enables the proper irradiation time and the effect of repeated doses to be determined, can be obtained simultaneously. This means that the phototoxic risk of compounds can be evaluated more clearly. Thus, incorporating phototoxicity assessments can overcome the problems that have been faced by combined studies so far, and is expected to contribute to safety assessment in the pharmaceutical industry.

Conflict of interest---- The authors declare that there is no conflict of interest.

\section{REFERENCES}

Allen, J.E. (1993): Drug-induced photosensitivity. Clin. Pharm., 12, 580-587.

Banerjee, P.S. and Ritschel, W.A. (1989): Transdermal permeation of vasopressin. I. Influence of $\mathrm{pH}$, concentration, shaving and surfactant on in vitro permeation. Int. J. Pharm., 49, 189-197.

Diffey, B.L., Daymond, T.J. and Fairgreaves, H. (1983): Phototoxic reactions to piroxicam, naproxen and tiaprofenic acid. Br. J. Rheumatol., 22, 239-242.

Draize, J.H. (1959): Dermal toxicity. In Appraisal of the safety of chemicals in foods, drugs and cosmetics. The Association of Food and Drug Officials of the United States, Texas State Department of Health, Austin, 46-59.

Elsherbiny, M.E., El-Kadi, A.O. and Brocks, D.R. (2008): The metabolism of amiodarone by various CYP isoenzymes of human and rat, and the inhibitory influence of ketoconazole. J. Pharm. Pharm. Sci., 11, 147-159.

Food and Drug Administration (2003): Guidance for Industry Photosafety Testing. Pharmacology Toxycology Coordinating Committee in the Centre for Drug Evaluation and Research (CDER) at the FDA.

Hamada, S., Sutou, S., Morita, T., Wakata, A., Asanami, S., Hosoya, S., Ozawa, S., Kondo, K., Nakajima, M., Shimada, H., Osawa, K., Kondo, Y., Asano, N., Sato, S., Tamura, H., Yajima, N., Marshall, R., Moore, C., Blakey, D.H., Schechtman, L.M., Weaver, J.L., Torous, D.K., Proudlock, R., Ito, S., Namiki, C. and Hayashi, M. (2001): Evaluation of the rodent micronucleus assay by a 28-day treatment protocol: Summary of the 13th Collaborative Study by the Collaborative Study Group for the Micronucleus Test (CSGMT)/Environmental Mutagen Society of Japan (JEMS)Mammalian Mutagenicity Study Group (MMS). Environ. Mol. Mutagen., 37, 93-110.

International Conference on Harmonisation of Technical Requirements for Registration of Pharmaceuticals for Human Use (2008): Genotoxicity Testing and Data Interpretation for Pharmaceuticals Intended for Human Use S2 (R1).

International Conference on Harmonisation of Technical Requirements for Registration of Pharmaceuticals for Human Use (2009): Guidance on nonclinical safety studies for the conduct of human clinical trials and marketing authorization for pharmaceuticals M3 (R2). In International conference on harmonisation of technical requirements for registration of pharmaceuticals for human use.

International Conference on Harmonisation of Technical Requirements for Registration of Pharmaceuticals for Human Use (2013): Photosafety Evaluation of Pharmaceuticals S10.

Luft, J. and Bode, G. (2002): Integration of safety pharmacology endpoints into toxicology studies. Fundam. Clin. Pharmacol., 16, 91-103.

Morikawa, F., Nakayama, Y., Fukuda, M., Hamano, M., Yokoyama, Y., Nagura, T., Ishihara, M. and Toda, K. (1974): Techniques for evaluation of phototoxicity and photoallergy in laboratory animals and man. In Sunlight and Man (T.B. Fitzpatrick, Ed.), pp. 529-557. University of Tokyo Press, Tokyo.

Morimura, T., Seki, T., Toyoizumi, T., Takaoka, Y. and Saito, Y. (2010): Oral dose phototoxicity study in rats: a study of combined phototoxicity and photochemical skin micronucleus test method. Annual Report of Hatano Research Institute, 33, 32-38.

Parrish, J.A., Fitzpatrick, T.B., Tanenbaum, L. and Pathak, M.A. (1974): Photochemotherapy of psoriasis with oral methoxsalen 
A general toxicity study incorporating phototoxicity assessments

and longwave ultraviolet light. N. Engl. J. Med., 291, 12071211.

Seto, Y., Kato, M., Yamada, S. and Onoue, S. (2013): Development of micellar reactive oxygen species assay for photosafety evaluation of poorly water-soluble chemicals. Toxicology In Vitro, 27, $1838-1846$.

Tice, R.R., Agurell, E., Anderson, D., Burlinson, B., Hartmann, A., Kobayashi, H., Miyamae, Y., Rojas, E., Ryu, J.C. and Sasaki, Y.F. (2000): Single cell gel/comet assay: guidelines for in vitro and in vivo genetic toxicology testing. Environ. Mol. Mutagen., 35, 206-221.

Türck, D., Busch, U., Heinzel, G. and Narjes, H. (1997): Clinical pharmacokinetics of meloxicam. Arzneimittelforschung, 47, 253-258.

Yonezawa, Y., Ohsumi, T., Miyashita, T., Kataoka, A., Hashimoto, K., Nejishima, H. and Ogawa, H. (2015). Evaluation of skin phototoxicity study using SD rats by transdermal and oral administration. J. Toxicol. Sci., 40, 667-683. 